\title{
Modeling and Analysis for Streaming Service Systems
}

\author{
Hai-Peng Zhang Bao-Qun Yin Xiao-Nong Lu \\ Department of Automation, University of Science and Technology of China, Hefei 230026, China
}

\begin{abstract}
The proliferation of streaming service system in various application areas gains increasing importance and also poses more challenges in the research of streaming service system. In this paper, we propose a novel dynamic model composed of a set of differential equations to describe the evolution of streaming service systems. And in the model, we focus on how the policies for admission control and peer selection influence on the system. We first introduce a flexible abstraction of streaming service systems. The abstraction is generally enough to capture the essences of streaming service systems with different structures, physical characteristics, software protocols and client behaviors. Then, by analyzing the state which is defined as the number of requests, a novel dynamic model is developed in microscopic scale to characterize the behaviors of streaming service systems. The model proposed in this paper demonstrates the interactions between clients and servers and also between different servers. The interactions are primarily influenced by the admission control policy and peer selection policy. Finally, some experiments are designed to verify the validation and reasonability of the model.
\end{abstract}

Keywords: Peer-to-peer, streaming service system, dynamic model, admission control policy, peer selection policy.

\section{Introduction}

Although streaming service systems were widely used in various application areas, such as entertainment, education and medical treatment in the last decade ${ }^{[1,2]}$, the development of streaming systems with high definition still faces numerous challenges. In order to improve quality of service (QoS), reduce running cost and lessen the traffic of backbone networks, the peer-to-peer $(\mathrm{P} 2 \mathrm{P})$ and content delivery network $(\mathrm{CDN})$ techniques are widely used in the streaming service systems ${ }^{[3,4]}$. By moving services from central servers to the proxy servers that are distributed on the edge of the internet, the streaming service system can quickly respond to the clients' requests. The proxy servers share resources with each other to meet the clients' ${ }^{\prime}$ demands for services not owned locally. The system makes full use of network resources, reduces investment cost and supplies better service to clients via the interactions between servers.

Since the study on streaming service system is very important, numerous researches have been done in various approaches. Three methods, which are often used in the study on streaming service system, are network measurement, network simulation, theoretical modeling and analysis of network, respectively. Network measurement has become an important method to capture more information of network and the behavior of clients. The actual measurements provide real-life data for analyzing the system and check out the theoretical assumptions. Schulze and Mochalski ${ }^{[5]}$ from ipoque (a German traffic management and analysis company) analyzed internet traffic in different applications and different regions, and they also mentioned that the streaming applications accounted for a considerable proportion of the internet traffic and attracted much attention. By analyzing the traces collected from a popular video-on-demand

\footnotetext{
Regular paper

Manuscript received June 8, 2013; revised September 11, 2013

This work was supported by National Natural Science Foundation of China (Nos. 61174124 and 61233003), Research Fund for Doctora Program of Higher Education of China (No. 20123402110029), and Natural Science Research Program of the Anhui High Education Bureau of China (No. KJ2012A286).
}

(VoD) system, Luo et al. ${ }^{[6]}$ pointed out that the popularity of video content is determined by various factors, such as the length of video, content type-different parts of the same video may have different popularities ${ }^{[6]}$. Furthermore, Zink et al. ${ }^{[7]}$ showed that different user populations may have different preferences of video content. Numerous measurements ${ }^{[7,8]}$ showed that most clients focus on a limited number of video objects, which supported the idea that the popularity follows a Zipf or Zipf-like distribution ${ }^{[9]}$ Lots of designs and optimizations of streaming service systems were based on the assumption that the popularity follows a Zipf or Zipf-like distribution, especially the caching management of the proxy servers. By caching the most popular video contents on the proxy servers, the system can reduce the demanding delay and lessen traffic of the backbone network ${ }^{[10,11]}$.

The data collected by the actual measurement is precise and convincing, but network measurement faces lots of challenges, such as the intricacy of technique, long-term tracing, high cost, etc. However, network simulator provides a novel method to obtain credible synthetic trace. Yang and Abu-Ghazaleh ${ }^{[12]}$ presented a network simulatorgeneral peer-to-peer simulator(GPS), which was aimed to accurately capture the features of BitTorrent, but the simulator has a poor extendibility. In contrast, the network simulator $2(\mathrm{NS}-2)^{[13]}$ with a high extendibility was widely used in simulating scenarios with various protocols, applications, and networks. Lots of researchers ${ }^{[14,15]}$ gained data to verify their studies on distinct networks by using NS-2.

But as was mentioned by Naicken et al. in their work ${ }^{[16]}$, the steep learning curve of NS-2 brought difficulties in application.

The modeling and analysis of network can offer theoretical support for the design and implementation of network architectures, protocols and applications. The dynamic differential equation, as one of the many ways to do theoretical modeling and analysis, reflects the evolutionary process of the object of focus and reveals the interactions between factors that influence the object. In Misra et al.'s work ${ }^{[17]}$, a 
dynamic model for transmission control protocol (TCP) flow was presented, and it captured the behavior of TCP throughput with a fair degree of accuracy. Qiu and Srikant ${ }^{[18]}$ proposed a fluid model for a BitTorrent-like system in a global view. The model described the evolvement of the number of peers of different types. Qiu and Sang ${ }^{[19]}$ analyzed the global stability of the model in their work, and concluded that the number of downloaders and seeds will reach a stable state after a finite time. However, in the model, the assumption that the arrival and the departure processes of downloaders followed a Poisson distribution, was in obvious contradiction with Pouwelse's measurement which showed that Qiu's assumption was not necessarily true, and the stable state can be reached but it may only last a short time ${ }^{[20]}$. Wu et al. ${ }^{[21]}$ adopted queueing network with infinite queues to model the multi-channel P2P live streaming system, which may not be used in real systems for the assumption that the capacity of server is unlimited. Zhou et al. ${ }^{[22]}$ derived the relationship between storage capacity, the number of videos, the number of peers and the bandwidth in their work, and proposed a replication algorithm to balance the average load. Zhou et al. ${ }^{[23]}$ proposed a chunk-scheduling model which mixed the rarest first and greedy policies for $\mathrm{P} 2 \mathrm{P}$ streaming systems to achieve the scalability and urgency in their work.

In our previous work ${ }^{[24,25]}$, we defined the state of system in a micro-view which is different from Qiu's work ${ }^{[18]}$. In our work ${ }^{[24]}$, we developed a dynamic model for the P2Pbased media delivery network (MDN) system. And in the model the state, which is defined as the amount of required data that has not been served from one node to another, is influenced by peer selection policy and bandwidth allocation policy. We also proposed another model for BitTorrentlike P2P file-sharing system in our work ${ }^{[25]}$. In the model, the state of one node is defined as the amount of required data that has not been served by the node, and the state is influenced by peer selection policy, bandwidth allocation policy, peer choking policy and piece selection policy.

The work in this paper adopts the method of theoretical modeling and analysis, which is quite different from the other methods of network measurement ${ }^{[5-8]}$ and network simulation ${ }^{[12-15]}$. The method of theoretical modeling and analysis reduces the consumption, makes full use of the conclusion founded by the other methods and offers a more intuitionistic expression in a mathematical way. The model in Misra et al.'s work ${ }^{[17]}$ presented a quite good method to describe TCP, but it had poor applicability. Qiu and Srikant ${ }^{[18]}$ modeled the P2P file-sharing system in a globalview in their work, but they ignored too much details of networks, e.g., the influence of algorithms and protocols on the networks. And the model they presented relied too much on some assumptions. The assumption and the computational complexity may lead to the fact that the model in Wu et al.'s work ${ }^{[21]}$ can not be used in real systems. Zhou et al. ${ }^{[22,23]}$ paid attention to one respect of the system, such as replication and chunk-scheduling in their work, but they ignored the relationship between different algorithms. This paper models the streaming service system in a micro-view, which focus on the states of one node and the factors that influence the node. Our previous work ${ }^{[24,25]}$ focused too much on the description of the details of networks, so it has good expansibility only in P2P file-sharing systems, especially for BitTorrent. The model presented in this paper has a much better expansibility by adopting a flexible abstraction which is almost suitable for all system structures.

In this paper, a flexible abstraction is presented, which is generally enough to capture the essence of streaming service systems with different structures, physical characteristics, software protocols and client behaviors. In the abstraction, the system can be seen as a network composed of virtual nodes (VNs), each of which represents a service local area network (LAN). Based on the abstraction, the state of one $\mathrm{VN}$ is defined as the number of different clients' requests admitted by the VN. Then a novel model is proposed to describe the interactions between clients and servers and between servers. The model is influenced by the behavior of clients, hardware and software characteristics. This model especially puts emphasis upon admission control policy and peer selection policy. The admission control policy, which is widely used in streaming service systems for resource optimization $^{[26,27]}$, decides whether the request can be admitted by the server. With the popularity of mobile devices, the admission control is becoming increasingly important in wireless network research ${ }^{[28,29]}$. The peer selection policy, meanwhile, has a deep influence on load balancing in that it decides which nodes will receive the requests. The goal of our work is to propose a dynamic model composed of a set of differential equations that can be used to illustrate each state of $\mathrm{VN}$. In the model, the relationship between states and some policies, such as admission control policy and peer selection policy, is discussed. Then the performance of the system can be analyzed and optimized based on the model.

The rest of this paper is organized as follows. In Section 2, a flexible abstraction of the system is presented. In Section 3, a dynamic model for streaming service system is developed. In Section 4, several specific policies for admission control and peer selection are analyzed and the concrete forms of the model proposed in Section 3 are given. In Section 5, some simulations are designed to prove the validation and reasonability of the model. Finally, Section 6 draws the conclusions.

\section{System structure}

A streaming service system is composed of numerous servers and much more clients. Different streaming service systems may have different structures, physical characteristics, software protocols and client behaviors. For instance, there may be various types of servers with different functions in the streaming service systems, such as manager servers, content servers, log servers, directory servers, proxy servers and so on. Although there may be many differences among various streaming service systems, the main processes of the systems, which are managing clients' requests and rendering services to clients, are much the same with each other. For ease of exposition, an abstraction, which is generally enough to capture the essence of streaming service systems, is presented in Fig. 1. The functions of each module in Fig. 1 are listed as below.

1) Admission control system is in charge of deciding whether the requests can be admitted. There may be three 
results: admission, rejection and transmission.

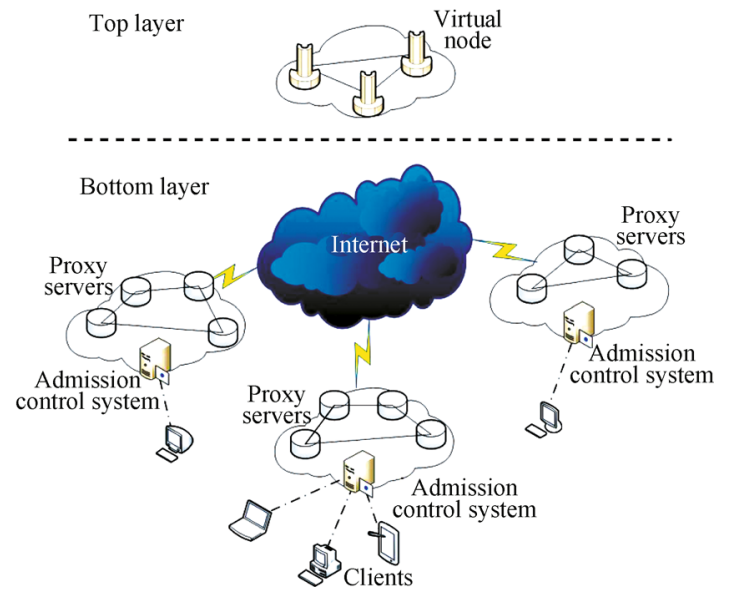

Fig. 1 The system structure of streaming service system

2) Proxy server renders services to clients. The proxy servers are located closely to the clients within the same LAN (or local clients). The limitation of servers' capacity leads to the rejection and transmission of the requests.

3) VN represents a service LAN which is composed of an admission control system and numerous proxy servers.

4) Clients send request to the local service subsystem represented by $\mathrm{VN}$ and enjoys service if the request is admitted.

The system can be regarded as a two-layer network, where the top layer is composed of VNs which are the abstraction of the service LANs, while the bottom layer is composed of proxy servers. The $\mathrm{VN}$ receives requests from both local clients and remote clients, and may transmit some of the requests from local clients to other VNs. The requests from local clients face three kinds of decision results which are listed in Table 1 . Whereas the remote clients' requests which are transmitted by other VNs will either be admitted or rejected, but not transmitted. This is simply to avoid repeated transmission.

Table 1 Decision results for requests from local clients

\begin{tabular}{|c|c|}
\hline Decision result & Content \\
\hline Admission & $\begin{array}{l}\text { The request is admitted and will be served } \\
\text { by the local proxy servers. }\end{array}$ \\
\hline Rejection & $\begin{array}{l}\text { The local VN rejects to render a service to } \\
\text { the request. }\end{array}$ \\
\hline Transmission & $\begin{array}{l}\text { The local VN decides to transmit the } \\
\text { requests from local clients to other VNs. }\end{array}$ \\
\hline
\end{tabular}

The system structure proposed in this part is an abstraction for the streaming service system. It focuses on the interactions between clients and servers and also between different servers, and some auxiliary functions will be ignored or expressed unclearly. The system structure proposed in this part is generally enough to capture the essence of streaming service systems, and has a nice extendibility, which means that the system structure can be applied to various streaming service systems.

\section{A dynamic model}

The dynamic model for the streaming service system is inspired by the work $[17-19,24,25]$. In this paper, we base our analysis on the scenario shown in Fig. 2, where the interactions between $\mathrm{VN}$ and local clients and between VNs are illustrated. In the model, a set of differential equations are given to describe the state of each VN. In order to capture the characteristics of streaming service systems, some parameters and variables used in the equations are defined as follows.

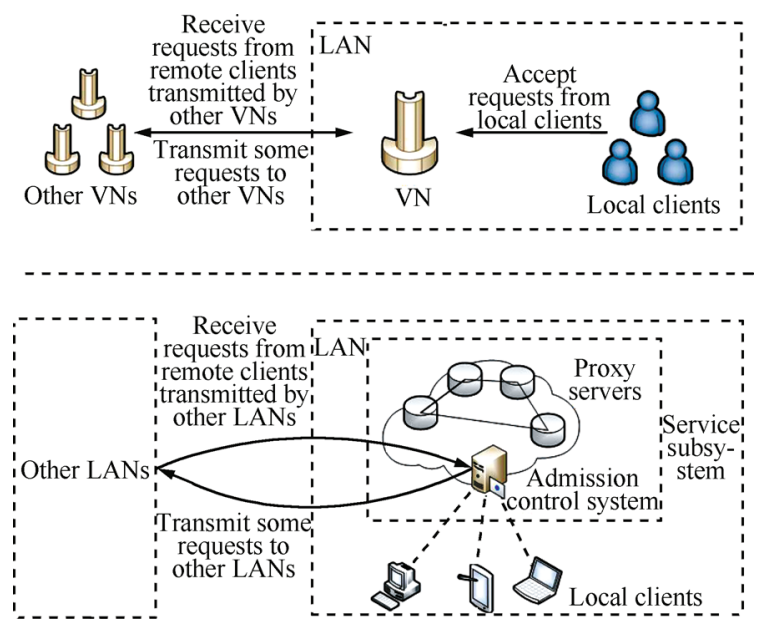

Fig. 2 Proxy-client network structure

$N$ : Number of VNs in the system.

$M$ : Number of services in the system.

$x_{i}(t)$ : Number of local clients' request admitted by $\mathrm{VN} i$ at time $t$.

$y_{i}(t)$ : Number of remote clients' requests transmitted by other $\mathrm{VNs}$ and admitted by $\mathrm{VN} i$ at time $t$.

$\lambda_{i m}$ : Arrival rate (number/per unit time) of local clients' requests received by $\mathrm{VN} i$ for service $m$.

$\mu_{i m}^{u}$ : Service rate (number/per unit time) of local clients' requests admitted by VN $i$ for service $m$.

$\mu_{i m}^{v}$ : Service rate (number/per unit time) of remote clients' requests transmitted by other VNs and admitted by $\mathrm{VN} i$ for service $m$.

$\boldsymbol{P}(t)$ : Service storage map at time $t, \boldsymbol{P}(t)=\left[p_{i j}(t)\right], 1 \leqslant$ $i \leqslant N, 1 \leqslant j \leqslant M$, in which $p_{i j}$ denotes whether VN $i$ has service $j$. If it does, $p_{i j}(t)=1$; otherwise, $p_{i j}(t)=0$.

The general model for the streaming service system will be introduced in the following part. The stability of VNs conduces to the invariance of $N$ and $M \cdot \boldsymbol{P}(t)$, which indicates the distribution of services, can also be assumed to be constant during a certain time period so that it can be denoted simply as $\boldsymbol{P}$. The arrival rate is a fundamental parameter describing clients' behaviors and can be easily obtained by measuring the number of requests received by $\mathrm{VN} i$ for service $m$ per unit time. Service rate, which is determined by clients' behavior and $\mathrm{VNs}^{\prime}$ capacity, can be obtained by measuring the number of services finished per unit time. Furthermore, the sums denoted by $\sum_{m=1}^{M} \mu_{i m}^{u}$ and $\sum_{m=1}^{M} \mu_{i m}^{v}$ reflect the decreasing rates of $x_{i}(t)$ and $y_{i}(t)$.

In the model, $f_{i m}^{u}\left(x_{1}, y_{1}, \cdots, x_{N}, y_{N}, \lambda_{i m}, p_{i m}\right)$ is defined as the arrival rate of local clients' requests admitted by $\mathrm{VN} i$ for service $m$ while $g_{i m}^{u}\left(x_{1}, y_{1}, \cdots, x_{N}, y_{N}, \lambda_{i m}, p_{i m}\right)$ is used to denote the arrival rate of local clients' requests transmitted by $\mathrm{VN} i$ for service $m$. They are determined by the arrival rate, current system state, and the admission 
control policy. Based on the previous definition and analysis, the admission process of requests from local clients is illustrated in Fig. 3 (a). Furthermore, the sum denoted by $\sum_{m=1}^{M} f_{i m}^{u}\left(x_{1}, y_{1}, \cdots, x_{N}, y_{N}, \lambda_{i m}, p_{i m}\right)$ reflects the increasing rate of $x_{i}(t)$.

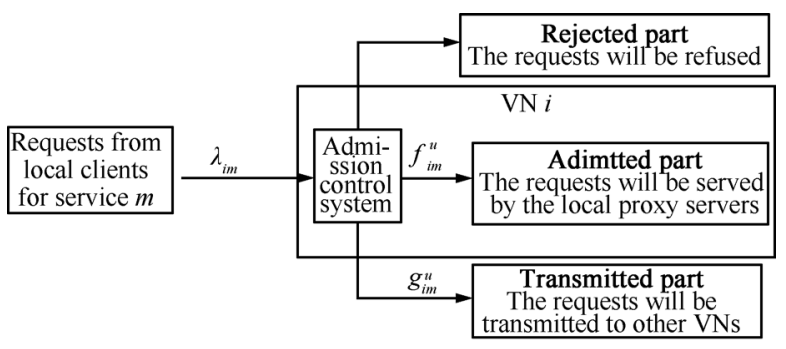

(a) Admission process of requests from local clients

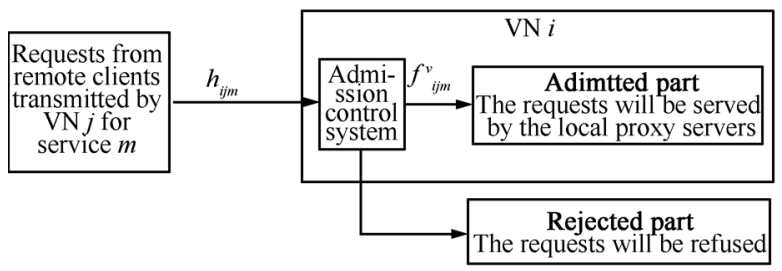

(b) Admission process of requests form remote clients

Fig. 3 Admission process of requests

$h_{i j m}$ is defined to denote the arrival rate of remote clients' $^{\prime}$ requests transmitted by $\mathrm{VN} j$ and received by $\mathrm{VN} i$ for service $m$, and is not only determined by clients' behaviors but also by the current system state, admission control policy, and peer selection policy. $f_{i j m}^{v}\left(x_{1}, y_{1}, \cdots, x_{N}, y_{N}, h_{i j m}, p_{i m}\right)$ is defined to denote the arrival rate of remote clients' requests transmitted by $\mathrm{VN}$ $j$ and admitted by $\mathrm{VN} i$ for service $m$. Based on the previous definitions and analysis, the admission process of requests from remote clients transmitted by other VNs is illustrated in Fig. 3 (b). Furthermore, the sum denoted by $\sum_{j=1, j \neq i}^{N} \sum_{m=1}^{M} f_{i j m}^{v}\left(x_{1}, y_{1}, \cdots, x_{N}, y_{N}, h_{i j m}, p_{i m}\right)$ reflects the increasing rate of $y_{i}(t)$.

Through the above analysis, a dynamic model for the evolution of the $\mathrm{VN}^{\prime}$ s state can be given by

$$
\left\{\begin{aligned}
\frac{\mathrm{d} x_{i}}{\mathrm{~d} t}= & -\sum_{m=1}^{M} \mu_{i m}^{u}+ \\
& \sum_{m=1}^{M} f_{i m}^{u}\left(x_{1}, y_{1}, \cdots, x_{N}, y_{N}, \lambda_{i m}, p_{i m}\right) \\
\frac{\mathrm{d} y_{i}}{\mathrm{~d} t}= & -\sum_{m=1}^{M} \mu_{i m}^{v}+ \\
& \sum_{j=1, j \neq i}^{N} \sum_{m=1}^{M} f_{i j m}^{v}\left(x_{1}, y_{1}, \cdots, x_{N}, y_{N}, h_{i j m}, p_{i m}\right) .
\end{aligned}\right.
$$

There are various policies for peer selection and admission control in streaming service systems, the detailed forms of the right-hand side of (1) cannot be obtained for general cases. Concrete forms of the right-hand sides of equations listed in (1) will be decided by the specific policies of peer selection and admission control.

\section{Analysis on the dynamic model}

Through the previous analysis, a dynamic model has been developed as shown in (1). Among all the policies in streaming service systems, peer selection policies and admission control policies have a significant influence on the system's performance. In this section, some specific policies of peer selection and admission control are introduced, followed by the corresponding concrete forms of the equations listed in (1).

\subsection{Analysis of basic policies}

Some basic peer selection and admission control policies that are independent of the states are introduced and analyzed in this part.

\subsubsection{Basic admission control policy}

The admission control policy is in charge of deciding whether the new arrived requests from both local and remote clients can be admitted. The basic admission control policy is that a $\mathrm{VN}$ admits all the requests if it has the corresponding services. Then the concrete forms of the components in the right-hand side of the equations in (1) are presented as

$$
\begin{aligned}
& f_{i m}^{u}\left(x_{1}, y_{1}, \cdots, x_{N}, y_{N}, \lambda_{i m}, p_{i m}\right)=\lambda_{i m} p_{i m} \\
& g_{i m}^{u}\left(x_{1}, y_{1}, \cdots, x_{N}, y_{N}, \lambda_{i m}, p_{i m}\right)=\lambda_{i m}\left(1-p_{i m}\right) \\
& f_{i j m}^{v}\left(x_{1}, y_{1}, \cdots, x_{N}, y_{N}, h_{i j m}, p_{i m}\right)=h_{i j m} p_{i m} .
\end{aligned}
$$

\subsubsection{Basic peer selection policy}

The peer selection policy decides which remote VNs will receive the requests from local clients transmitted by local $\mathrm{VN}$. The basic peer selection policy is that the remote VNs that have the requested service have the same chance to be selected. Then we will have the following result.

$$
h_{i j m}=\frac{p_{i m} g_{j m}^{u}}{\sum_{n=1}^{N} p_{n m}} .
$$

As shown in (2-5), the basic admission control and peer selection policies are independent of $\mathrm{VNs}^{\prime}$ states. The decisions on whether the requests can be admitted or which VNs will receive the transmitted requests are made just on the basis of the distribution of services.

\subsection{Analysis of the advanced policies}

Without considering the dynamic situation of the VNs, the basic admission control and peer selection policies in Section 4.1 are decided only by whether the VN has the requested service. To make up for this shortcoming, some advanced peer selection and admission control policies which are dependent on $\mathrm{VNs}^{\prime}$ states will be introduced and analyzed in the following part.

\subsubsection{State-based admission control policy}

The threshold-based admission control policy is a typical state-based policy. In a streaming service system, the capacity of a $\mathrm{VN}$ is usually referred to as the largest amount the requests that can be admitted. Consequently, requests that are beyond the capacity of a VN will either be transmitted to VNs in other LANs or simply rejected according to the threshold-based policy. However, the excess requests 
from remote clients will only be rejected by $\mathrm{VN}$, but not transmitted. Most of the admission control policies can be deemed as threshold-based in essence, with the difference between them being the way to set the capacity. The admission process of requests under threshold-based admission control policy is shown in Fig. 4. For ease of exposition, some parameters are defined as

$Q_{i}^{u}$ : Upper limit of the number of local clients' requests admitted by $\mathrm{VN} i$.

$Q_{i}^{v}$ : Upper limit of the number of remote clients' requests admitted by $\mathrm{VN} i$.

The values of $Q_{i}^{u}$ and $Q_{i}^{v}$ are different in different admission control policies, but they are usually decided by the capacity of VN. Then the concrete forms of the components in the right-hand side of the equations in (1) are presented as

$$
\begin{gathered}
f_{i m}^{u}\left(x_{1}, y_{1}, \cdots, x_{N}, y_{N}, \lambda_{i m}, p_{i m}\right)= \\
\lambda_{i m} p_{i m} I\left(Q_{i}^{u}-x_{i}\right) \\
g_{i m}^{u}\left(x_{1}, y_{1}, \cdots, x_{N}, y_{N}, \lambda_{i m}, p_{i m}\right)= \\
\lambda_{i m}\left(1-p_{i m}\right)\left(1-I\left(Q_{i}^{u}-x_{i}\right)\right) \\
f_{i j m}^{v}\left(x_{1}, y_{1}, \cdots, x_{N}, y_{N}, h_{i j m}, p_{i m}\right)= \\
h_{i j m} p_{i m} I\left(Q_{i}^{v}-y_{i}\right)
\end{gathered}
$$

where

$$
I(x)= \begin{cases}1, & \text { if } x>0 \\ 0, & \text { if } x \leqslant 0 .\end{cases}
$$

In (6) and (7), $I\left(Q_{i}^{u}-x_{i}\right)$ represents whether the number of local clients' requests admitted by $\mathrm{VN} i$ reaches the upper limit of $\mathrm{VN} i$

\subsubsection{State-based peer selection policy}

When a request from a local client is determined to be transmitted to other $\mathrm{VNs}$, the local VN must choose one $\mathrm{VN}$ in a source list provided by the system. According to its definition, the state of a VN reflects its working load. In most peer selection policies, the low-loaded VNs are more likely to be chosen as the target of request transmission than the high-loaded ones for the sake of loading balance. For ease of exposition, some parameters are defined as $s_{i}$ : Amount of requests admitted by $\mathrm{VN} i, s_{i}=x_{i}+y_{i}$.
$S_{m}$ : Amount of requests admitted by VNs that have service $m, S_{m}=\sum_{i=1}^{N} s_{i} p_{i m}$.

Then we will have the following result as

$$
h_{i j m}=\frac{p_{i m}\left(S_{m}-p_{i m} s_{i}\right)}{\sum_{n=1, n \neq j}^{N} p_{n m}\left(S_{m}-p_{n m} s_{n}\right)} g_{j m}^{u} .
$$

In the section above, the state-based admission control and peer selection policies are introduced and analyzed. And we can reach the conclusion based on what is shown in (6)-(8), (10) that the decisions on request admission and transmission are affected by the states of VNs and the distribution of services.

\section{Validation}

We will conduct a series of experiments to validate the dynamic model proposed in Sections 3 and 4. As is mentioned above, the states of VNs, which can also be deemed as the number of concurrent online clients, keep changing

\begin{tabular}{|c|c|}
\hline Condition & Content \\
\hline Condition 1 & $\begin{array}{l}\text { The capacities of VNs are large enough to } \\
\text { render services to all requests. }\end{array}$ \\
\hline Condition 2 & $\begin{array}{l}\text { The VNs do not have enough capacities to } \\
\text { render services to all requests, which } \\
\text { means that some requests cannot be answered, } \\
\text { at least not within a short time. }\end{array}$ \\
\hline
\end{tabular}
as the system's running condition varies over time. However, generally speaking, we can classify the system's various running conditions into two types listed in Table 2. In this section, a series of experiments are designed to validate the dynamic model under different running conditions.

Table 2 Two types of streaming service system's running conditions

\section{$5.1 \quad$ Experiment 1}

In the scenario, we simulate the evolvement of the streaming service system under the first running condition in Table 2. Since one streaming service system with several

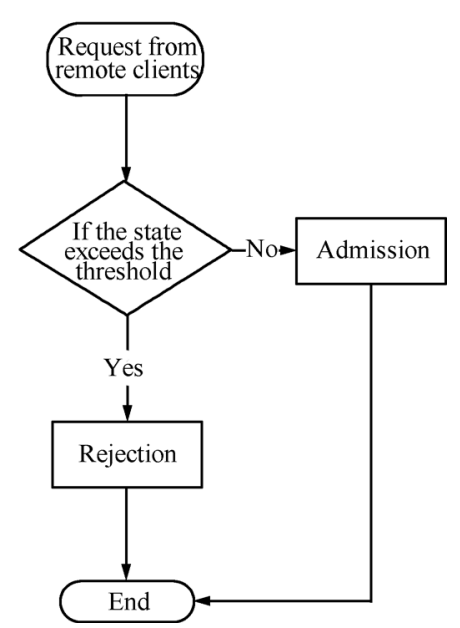

Fig. 4 Admission process of requests under threshold-based admission control policy 
VNs is generally enough to validate the model under this running condition, we can simplify the simulation process by narrowing down the subjects of analysis to two VNs, which are denoted as VN1 and VN2, and four services in the system. The service storage map $\boldsymbol{P}$ is set as

$$
\boldsymbol{P}=\left[\begin{array}{llll}
1 & 1 & 0 & 1 \\
0 & 1 & 1 & 1
\end{array}\right]
$$

The arrival rates of requests for different services are set as

$$
\left\{\begin{array}{l}
\lambda_{11}=20, \lambda_{12}=10, \lambda_{13}=8, \lambda_{14}=4 \\
\lambda_{21}=20, \lambda_{22}=10, \lambda_{23}=8, \lambda_{24}=4
\end{array}\right.
$$

And the service time of a different service follows exponential distribution with a different rate parameter. $\gamma_{i}$ is used to denote the rate parameter of service $i$, then $\gamma_{1}=0.25, \gamma_{2}=0.2, \gamma_{3}=0.125$, and $\gamma_{4}=0.1$. In this scenario, we adopt the basic policies of admission control and peer selection mentioned in Section 4.1. The theoretical values of $x_{1}$ and $y_{1}$ can be got by (1)-(5). The processes in which the clients enter and leave the system are simulated to get the information on the number of requests from both local and remote clients. As shown in Fig. 5, the simulation results match well with the theoretical values of $x_{1}$ and $y_{1}$. Furthermore, the number of requests admitted by VN will reach a stable state after a finite time in this scenario.

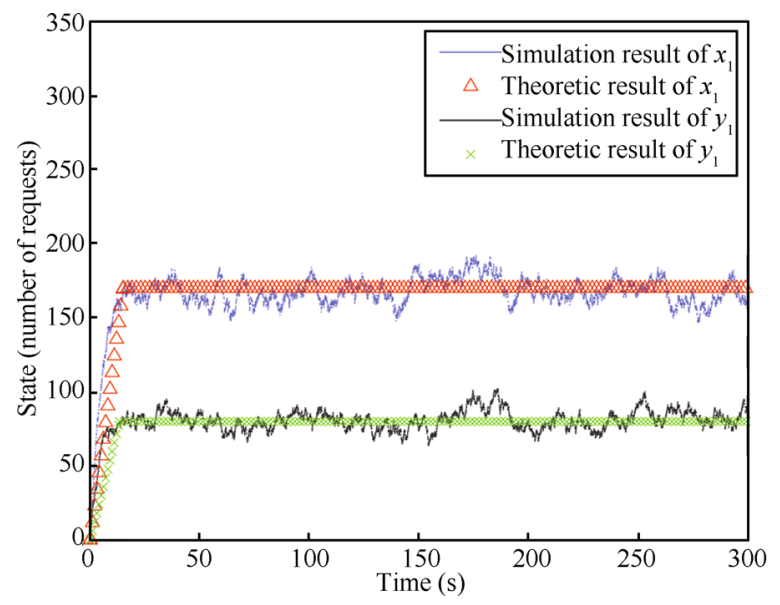

Fig. 5 Evolution of the state $x_{1}, y_{1}$ as a function of time

\subsection{Experiment 2}

In this scenario, we simulate the evolvement of the streaming service system under the second running condition in Table 2. We assume that the requests can not leave the system under the requested service is finished. One VN with one service is generally enough to demonstrate the evolution of the system under this running condition. We set the arrival rate $\lambda_{11}=10$ and the service time follows exponential distribution with the rate parameter as 0.1. The upper limit of the capacity of the $\mathrm{VN}$ is 80 , which means that the VN can only render services to no more than 80 requests concurrently. We measure the number of requests and compare the simulation results with the theoretic ones in Fig. 6. As shown in Fig. 6, adopting the basic admission control policy under the condition that the VN can not afford services to all requests leads to the continual growth of the number of requests, which may cause the system collapse.

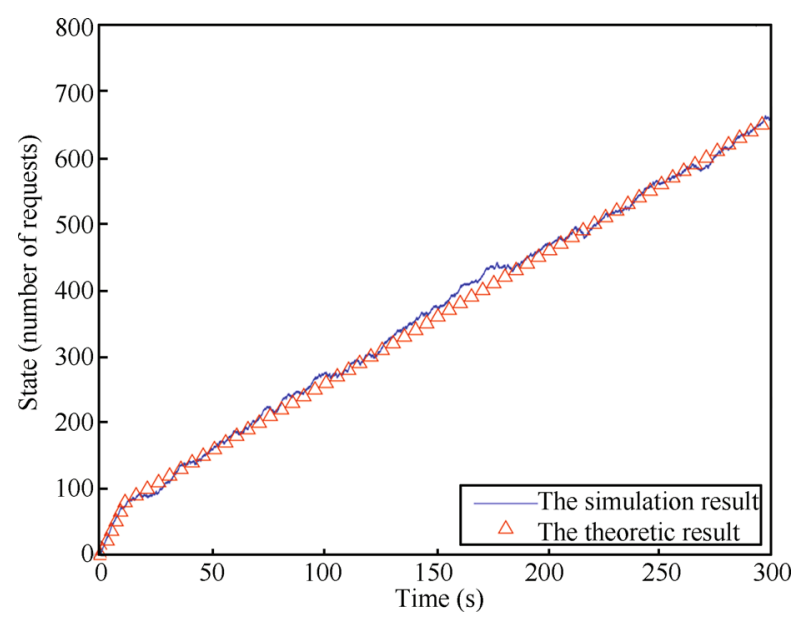

Fig. 6 Evolution of the state $x_{1}$ as a function of time

\subsection{Experiment 3}

In this scenario, we compare the basic peer selection policy and the state-based one under the second running condition in Table 2. We can simplify the simulation process by narrowing down the subjects of analysis to three VNs, which are denoted as PN1, PN2, and PN3, respectively. And there are four services in the system, then we set the service storage map $\boldsymbol{P}$ as

$$
\boldsymbol{P}=\left[\begin{array}{llll}
1 & 1 & 1 & 0 \\
0 & 1 & 0 & 1 \\
1 & 1 & 1 & 0
\end{array}\right]
$$

The arrival rates of requests for different services are set as

$$
\left\{\begin{array}{l}
\lambda_{11}=20, \lambda_{12}=10, \lambda_{13}=8, \lambda_{14}=4 \\
\lambda_{21}=10, \lambda_{22}=5, \lambda_{23}=4, \lambda_{24}=2 \\
\lambda_{31}=10, \lambda_{32}=5, \lambda_{33}=4, \lambda_{34}=2 .
\end{array}\right.
$$

And the service times of different services follow exponential distribution with different rate parameters. $\gamma_{i}$ is used to denote the rate parameter of service $i$, then $\gamma_{1}=0.25, \gamma_{2}=0.2, \gamma_{3}=0.125$, and $\gamma_{4}=0.1$. As shown in Figs. 7 and 8, the simulation results match well with the theoretical values. Loading ratio, which is defined as the ratio of the number of admitted requests to the upper limit of the VN, is often used to describe the loading balance of the system. Every $\mathrm{VN}$ is assumed to have a same upper limit 300. As shown in Fig. 9, the state-based policy can effectively reduce the loading of high-loaded VN by transmitting the requests to the low-loaded VNs.

\subsection{Experiment 4}

In this scenario, we compare the basic admission control policy and the state-based one under the second running condition in Table 2. One VN with one service is generally enough to demonstrate the evolution of the system under 


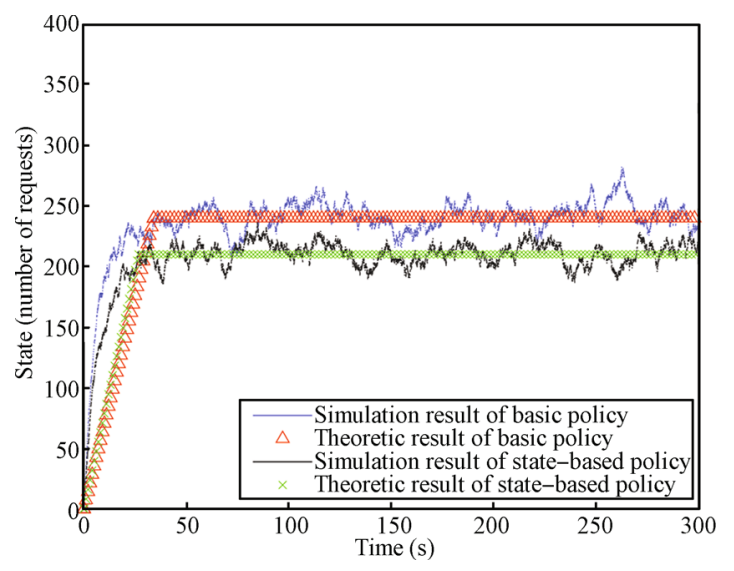

Fig. 7 Evolution of the state $x_{1}+y_{1}$ as the function of time

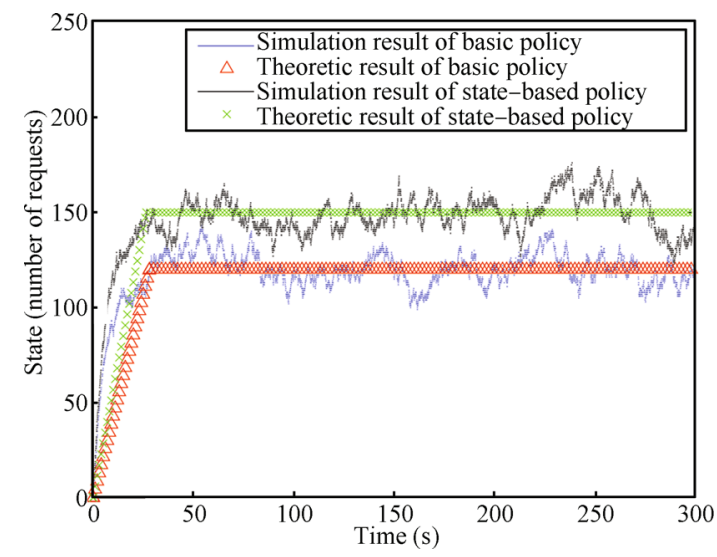

Fig. 8 Evolution of the state $x_{3}+y_{3}$ as the function of time

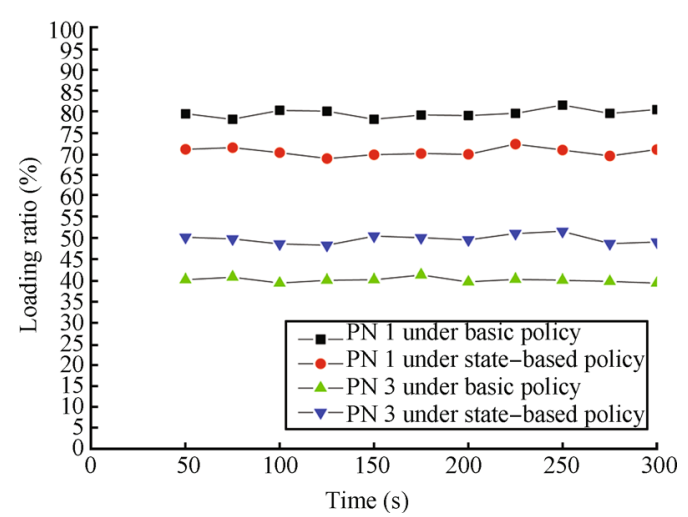

Fig. 9 Loading ration evolution comparison between basic policy and state-based policy

this running condition. We set the arrival rate $\lambda_{11}=10$ and the service time follows exponential distribution with the rate parameter 0.1 . The upper capacity limit of the $\mathrm{VN}$ is 80 , which means that the $\mathrm{VN}$ can only render services to no more than 80 requests concurrently. And we assume that the requests will not leave the system until the requested services are finished. As shown in Fig. 10, adopting the state-based admission policy can avoid the collapse of the system caused by the unceasing increase of requests.

Streaming service systems are always time-critical. Demanding delay, which is defined as the time interval between the moment when a request arrives at the server and the moment when the server starts to serve the request, is an important parameter in estimating a streaming service system. High-demanding-delay usually leads to the losing of customers. The demanding delay under this scenario is obtained to compare the basic policy with the state-based one. As shown in Fig. 11, the state-based policy can keep the demanding delay low by refusing services to the requests that are beyond the upper limit of the VNs while the demanding delay in the VNs adopting the basic admission control policy keeps increasing when the number of admitted requests exceeds the upper limits of the VNs.

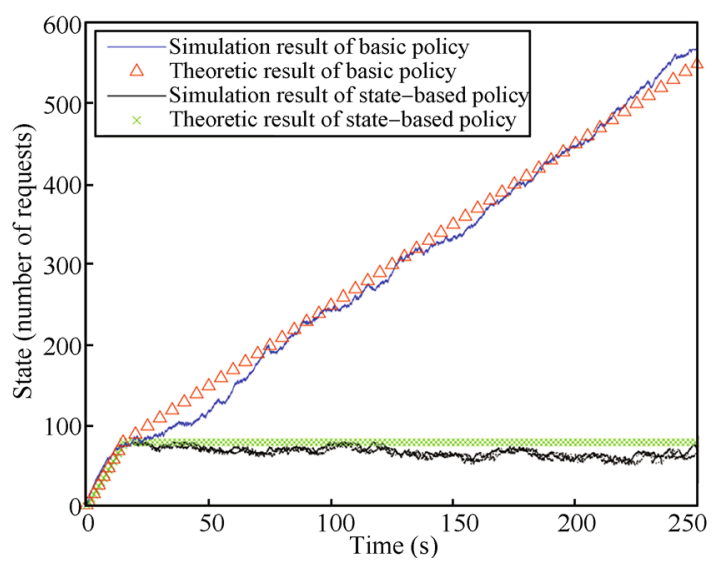

Fig. 10 Evolution of state $x_{1}$ as the function of time

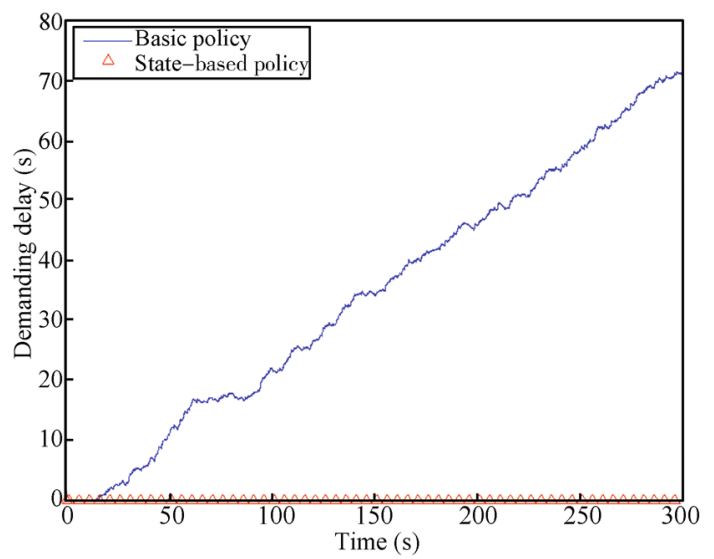

Fig. 11 Demanding delay evolution comparison between basic policy and state-based policy

\subsection{Experiment 5}

In this scenario, a system is designed and implemented to prove and compare the policies proposed in Section 4. We consider a system with five VNs denoted as VN1, VN2, VN3, VN4 and VN5, and there are four services in the system. The service storage map $\boldsymbol{P}$ is set as

$$
\boldsymbol{P}=\left[\begin{array}{llll}
1 & 1 & 0 & 1 \\
0 & 1 & 1 & 0 \\
0 & 1 & 0 & 1 \\
1 & 0 & 1 & 0 \\
1 & 1 & 1 & 0
\end{array}\right]
$$

For ease of exposition, the service times of different services follow the exponential distribution with different rate pa- 
rameters. $\gamma_{i}$ is used to denote the rate parameter of service $i$, then $\gamma_{1}=0.25, \gamma_{2}=0.2, \gamma_{3}=0.125$, and $\gamma_{4}=0.1$. The experiments are done under two conditions denoted as $C 1$ and $C 2$, in which the arrival rates are different. The arrival rates are set as follows.

$$
\begin{gathered}
C 1:\left\{\begin{array}{l}
\lambda_{11}=20, \lambda_{12}=10, \lambda_{13}=8, \lambda_{14}=4 \\
\lambda_{21}=15, \lambda_{22}=7.5, \lambda_{23}=6, \lambda_{24}=3 \\
\lambda_{31}=15, \lambda_{32}=7.5, \lambda_{33}=6, \lambda_{34}=3 \\
\lambda_{41}=15, \lambda_{42}=7.5, \lambda_{43}=6, \lambda_{44}=3 \\
\lambda_{51}=20, \lambda_{52}=10, \lambda_{53}=8, \lambda_{54}=4
\end{array}\right. \\
C 2:\left\{\begin{array}{l}
\lambda_{11}=25, \lambda_{12}=15, \lambda_{13}=10, \lambda_{14}=8 \\
\lambda_{21}=20, \lambda_{22}=10, \lambda_{23}=8, \lambda_{24}=5 \\
\lambda_{31}=20, \lambda_{32}=10, \lambda_{33}=8, \lambda_{34}=5 \\
\lambda_{41}=20, \lambda_{42}=10, \lambda_{43}=8, \lambda_{44}=5 \\
\lambda_{51}=25, \lambda_{52}=15, \lambda_{53}=10, \lambda_{54}=8 .
\end{array}\right.
\end{gathered}
$$

The state-based admission control policy proposed in Section 4.2.1 is adopted in this scenario. Then the upper limits of the VNs are set as

$$
\left\{\begin{array}{l}
Q_{1}^{u}=200, Q_{2}^{u}=100, Q_{3}^{u}=100, Q_{4}^{u}=100, Q_{5}^{u}=200 \\
Q_{1}^{v}=100, Q_{2}^{v}=50, Q_{3}^{v}=50, Q_{4}^{v}=50, Q_{5}^{v}=100 .
\end{array}\right.
$$

The arrival rates in $C 1$ are much smaller than those in $C 2$. High arrival rates lead to the high loading. As shown in Figs. 12-15, the system under $C 2$ has a much higher loading than that under $C 1$. Admission ratio, which is defined as the ratio of number of admitted requests to the number of requests received by the system, is often used to estimate a streaming service system. We compare the admission ratios of the system adopting basic or state-based policies under different conditions in Fig. 16. As shown in Fig. 16, the state-based policies can effectively improve admission ratio by transmitting the loading of high-loaded VNs to the low-loaded ones.

\section{Conclusions}

In this paper, we present a novel dynamic model composed of a set of differential equations for the streaming service system. Based on an abstraction in which a streaming service system can be seen as one composed of several $\mathrm{VNs}$, a novel definition of the $\mathrm{VN}^{\prime} \mathrm{s}$ state is given as the number of requests admitted by the VN. Through analyzing the admission control and peer selection policies that influence the $\mathrm{VN}^{\prime}$ s state, a set of differential equations are given to capture the characteristics of the system. Some
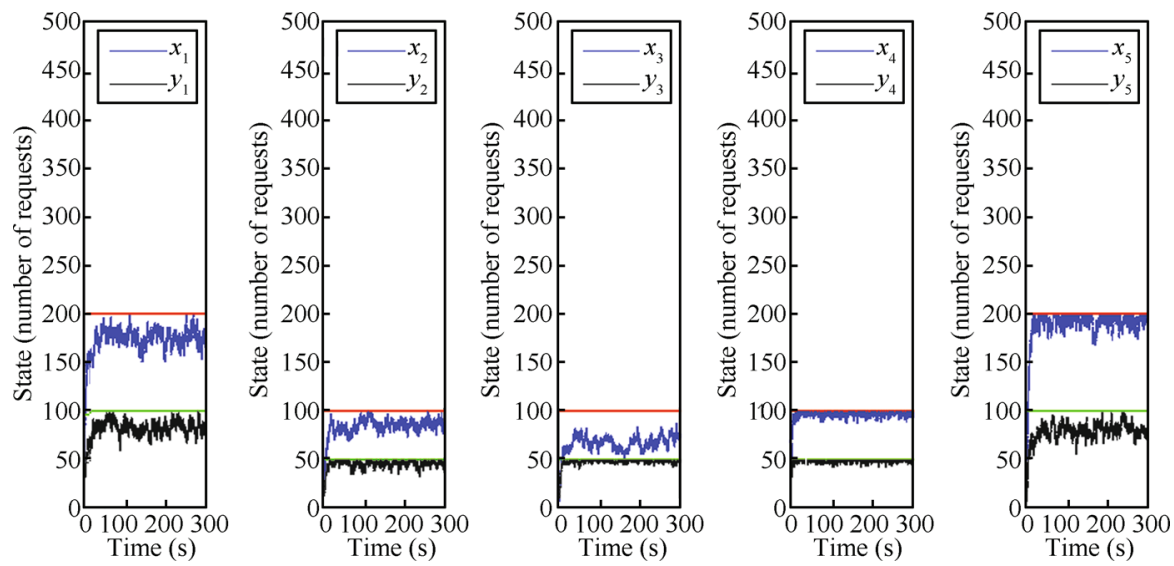

Fig. 12 Evolution of the system adopting basic policies under $C 1$
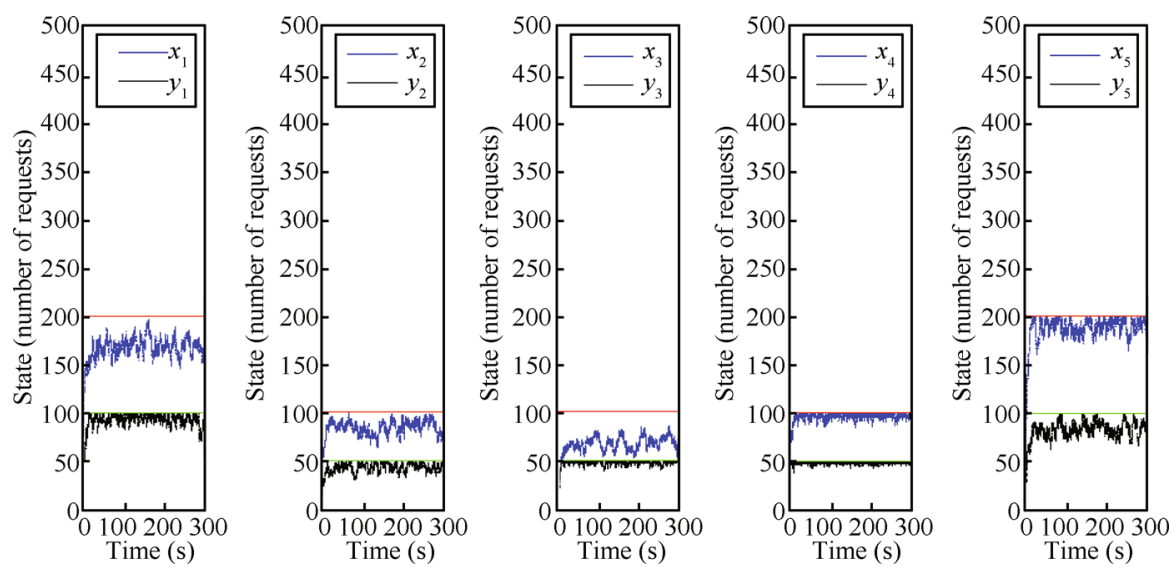

Fig. 13 Evolution of the system adopting state-based policies under $C 1$ 

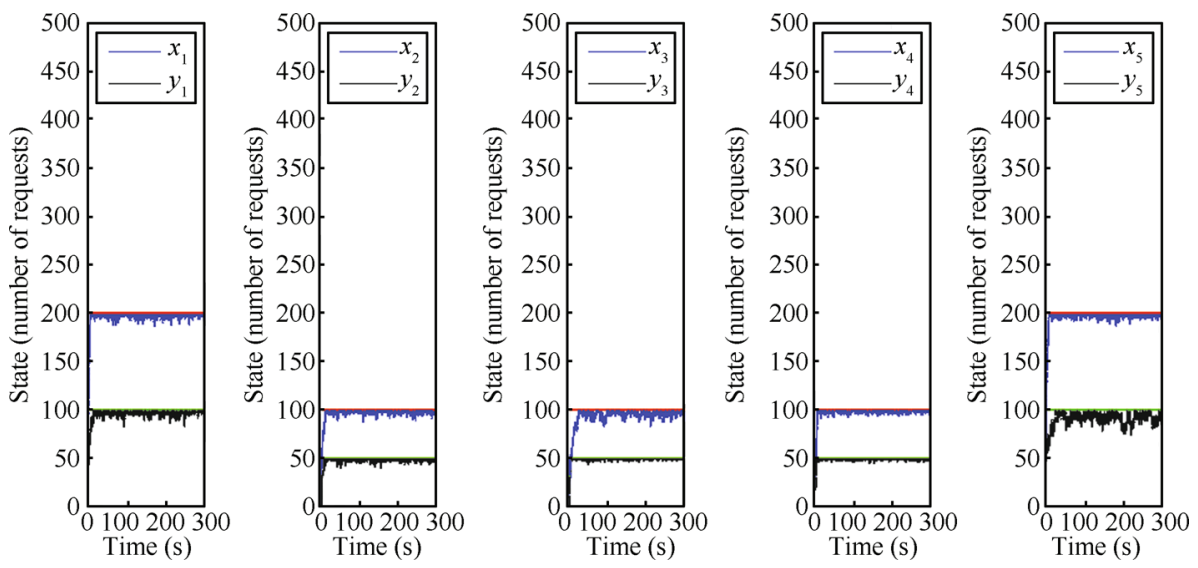

Fig. 14 Evolution of the system adopting basic policies under $C 2$
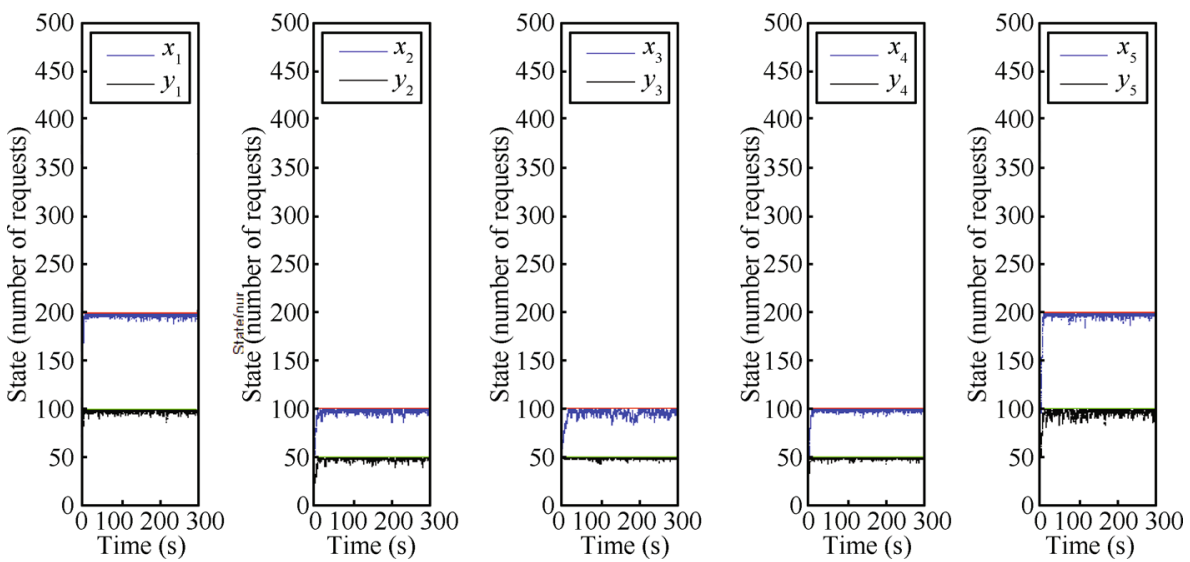

Fig. 15 Evolution of the system adopting state-based policies under $C 2$

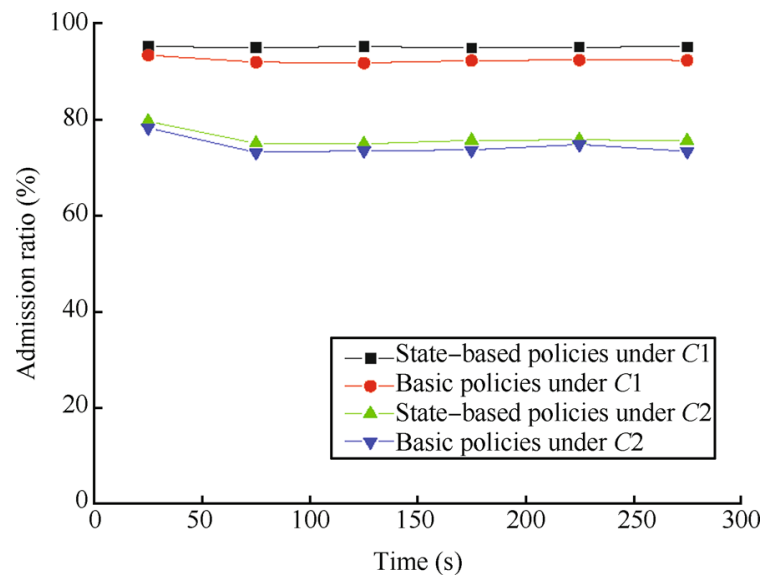

Fig. 16 Admission ratios for basic policies and state-based policies

specific policies of peer selection and admission control are introduced, followed by the corresponding concrete forms of the equations in the model. Then a series of simulations are designed to imitate the performance of the streaming service system under different running conditions, and are used to validate the dynamic model. The results of these simulations show that the model can precisely capture the characteristics of streaming service systems. Besides, the state-based policies have a better influence on the system's performance than the basic ones. The dynamic model proposed in this paper provides a novel method for analysis and optimization of the streaming service system. It can offer theoretical support for the design and implementation of network architectures, protocols and applications.

\section{References}

[1] W. Chen, C. C. Shih. Architecture of portable electronic medical records system integrated with streaming media. Journal of Medical Systems, vol. 36, no. 1, pp. 25-31, 2012.

[2] F. Zhang, W. H. Sun, Y. X. Huo. P2P streaming media technology in the remote education system. Advanced Materials Research, vol. 433-440, pp. 4893-4897, 2012.

[3] S. Borst, V. Gupta, A. Walid. Self-organizing algorithms for cache cooperation in content distribution networks. Bell Labs Technical Journal, vol. 14, no. 3, pp. 113-125, 2009.

[4] L. Guo, S. Q. Chen, X. D. Zhang. Design and evaluation of a scalable and reliable P2P assisted proxy for on-demand streaming media delivery. IEEE Transactions on Knowledge and Data Engineering, vol. 18, no. 5, pp.669-682, 2006.

[5] H. Schulze, K. Mochalski. Internet Study 2008/2009, [Online], Available: http://www.ipoque.com/s-ites/default/ files/mediafiles/documents/internet-study-20-08-2009.pdf, March 15, 2013.

[6] J. G. Luo, Q. Zhang, T. Tang, S. Q. Yang. A trace-driven approach to evaluate the scalability of P2P-based video-ondemand service. IEEE Transactions on Parallel and Distributed Systems, vol. 20, no. 1, pp. 59-70, 2009 
7] M. Zink, K. Suh, Y. Gu, J. Kurose. Characteristics of YouTube network traffic at a campus networkmeasurements, models, and implications. Computer Networks, vol. 53, no. 4, pp. 501-514, 2009.

[8] S. Acharya, B. Smith, P. Parnes. Characterizing user access to videos on the world wide web. In Proceedings of IS \& T/SPIE Multimedia Computing and Networking (MMCN2000), IS\&T/SPIE, San Jose, USA, pp.130-141, 2000.

[9] G. K. Zipf. Human Behavior and the Principle of Least Effort, Reading, MA, UK: Addison-Wesley, 1949.

[10] J. Yu, C. T. Chou, Z. K. Yang, X. Du, T. Wang. A dynamic caching algorithm based on internal popularity distribution of streaming media. Multimedia Systems, vol. 12, no. 2, pp. 135-149, 2006.

[11] T. Fujimoto, R. Endo, K. Matsumoto, H. Shigeno. Videopopularity-based caching scheme for P2P video-on-demand streaming. In Proceedings of IEEE International Conference on Advanced Information Networking and Applications, IEEE, Singapore, pp. 748-755, 2011.

[12] W. S. Yang, N. Abu-Ghazaleh. GPS: a general peer-to-peer simulator and its use for modeling BitTorrent. In Proceedings of the 13th IEEE International Symposium on Modeling Analysis, and Simulation of Computer and Telecommunication Systems, IEEE, Atlanta, USA, pp. 425-432, 2005.

[13] K. Fall, K. Varadhan. The Network Simulator ns-2. [Online], Available: http://www.isi.edu/nsnam/ns, March 15, 2013.

[14] M. S. Hasan, C. Harding, H. N. Yu, A. Griffiths. Modeling delay and packet drop in networked control systems using network simulator NS2. International Journal of Automation and Computing, vol. 2, no. 2, pp. 187-194, 2005.

[15] J. M. M. Kamal, M. S. Hasan, A. L. Griffiths, H. N. Yu. Development and verification of simulation model based on real MANET experiments for transport layer protocols (UDP and TCP). International Journal of Automation and Computing, vol. 10, no. 1, pp. 53-63, 2013.

[16] S. Naicken, B. Livinston, A. Basu, S. Rodhetbhai, I. Wakeman, D. Chalmers. The state of peer-to-peer simulators and simulations. In Proceedings of ACM SIGCOMM Computer Communication Review, ACM, New York, USA, vol.37, no. 2, pp. 95-98, 2007.

[17] V. Misra, W. Gong, D. Towsley. Stochastic differential equation modeling and analysis of TCP windowsize behavior. In Proceedings of Performance, Istanbul, Turkey, [Online], Available: http://citeseerx.ist.psu.ed$\mathrm{u} /$ viewdoc/download?doi=10.1.1.45.9562\&rep=rep1\&type$=$ pdf, March 15, 2013.

[18] D. Y. Qiu, R. Srikant. Modeling and performance analysis of BitTorrent-like peer-to-peer networks. In Proceedings of ACM SIGCOMM Computer Communication Review, ACM, New York, USA, pp. 367-378, 2004.

[19] D. Y. Qiu, W. Q. Sang. Global stability of peer-to-peer file sharing systems. Computer Communications, vol. 31, no. 2, pp. 212-219, 2008.

[20] J. Pouwelse, P. Garbacki, D. Epema, H. Sips. The Bittorrent P2P file-sharing system: Measurements and analysis. In Proceedings of the 4th International Conference on Peer-to-Peer Systems, Springer-Verlag, Berlin, Heidelberg, pp. 205-216, 2005.

[21] D. Wu, Y. Liu, K. W. Ross. Queuing network models for multi-channel P2P live streaming systems. In Proceedings of INFOCOM 2009, IEEE, Rio de Janeiro, Brazil, pp. 73$81,2009$.

[22] Y. P. Zhou, T. Z. J. Fu, D. M. Chiu. Statistical modeling and analysis of P2P replication to support VoD service. In Proceedings of INFOCOM 2011, IEEE, Shanghai, China, pp. 945-953, 2011.
[23] Y. P. Zhou, D.-M. Chiu, J. C. S. Lui. A simple model for chunk-scheduling strategies in P2P streaming. IEEE/ACM Transactions on Networking, vol. 19, no. 1, pp. 42-54, 2011.

[24] B. Q. Yin, D. Guo, J. Huang, X. M. Wu. Modeling and analysis for the P2P-based media delivery network. Mathematical and Computer Modelling, vol. 55, no. 3-4, pp. 1529$1539,2011$.

[25] H. P. Zhang, B. Q. Yin, X. N. Lu. A dynamic model of BitTorrent-like P2P file-sharing system. In Proceedings of the 31st Chinese Control Conference, IEEE, Hefei, China, pp. 5513-5517, 2012.

[26] B. Q. Yin, S. Lu, D. Guo. Analysis of admission control in P2P-based media delivery network based on POMDP. International Journal of Innovative Computing Information and Control, vol. 7, no. 7B, pp. 4411-4422, 2011.

[27] F. S. Lin, B. Q. Yin, J. Huang, X. M. Wu. Admission control with elastic QoS for video on demand systems. International Journal of Automation and Computing, vol. 9, no. 5, pp. 467-473, 2012.

[28] F. Zarai, K. B. Ali, M. S. Obaidat, L. Kamoun. Adaptive call admission control in 3GPP LTE networks. International Journal of Communication Systems, 2013. (Online first).

[29] D. H. Kim, D. L. Zhang, N. Bhushan, R. Pankaj, S. J. Oh. Admission control for cellular networks with direct QoS monitoring. Wireless Networks, vol. 19, no. 2, pp.131-144, 2013.

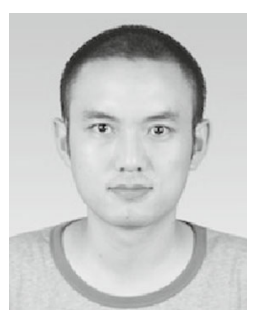

Hai-Peng Zhang received his B. Eng. degree in automation from University of Science and Technology of China, China in 2009 , and is currently a Ph. D. candidate in Department of Automation at University of Science and Technology of China.

His research interests include modeling, optimization and performance analysis of the networking.

E-mail: zhpeng@mail.ustc.edu.cn

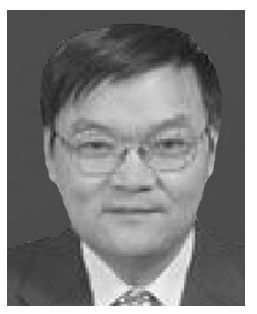

Bao-Qun Yin received his B. Sc. degree in fundamental mathematics from Sichuan University, China in 1985, his M. Sc. degree in applied mathematics from University of Science and Technology of China, China in 1993, and his $\mathrm{Ph} . \mathrm{D}$. degree in pattern recognition and intelligent system from University of Science and Technology of China, China in 1998. He is currently a professor of control science and engineering at Department of Automation, University of Science and Technology of China.

His research interests include discrete event dynamic systems, Markov decision processes, queuing systems, and network resource management and optimization.

E-mail: bqyin@ustc.edu.cn (Corresponding author)

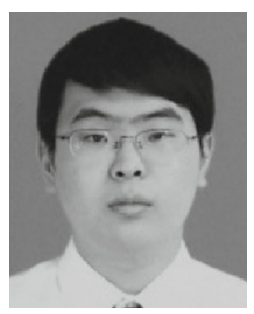

Xiao-Nong Lu received his B. Sc. degree in automation from University of Science and Technology of China, China in 2010. He is currently a Ph. D. candidate in School of Information Science and Technology, University of Science and Technology of China.

His research interests include discrete stochastic dynamic programming, analysis and optimization of admission control in streaming service system.

E-mail: lxn520@mail.ustc.edu.cn 